\title{
Detecting candidates of depression, anxiety and stress through malay-written tweets: a preliminary study
}

\author{
Muhammad Zahier Nasrudin ${ }^{1}$, Ruhaila Maskat ${ }^{2}$, Ramli Musa ${ }^{3}$ \\ ${ }^{1,2}$ Faculty of Computer \& Mathematical Sciences, Universiti Teknologi MARA, Malaysia \\ ${ }^{3}$ Department of Psychiatry, Kulliyyah of Medicine, International Islamic University Malaysia, Malaysia
}

\begin{tabular}{l}
\hline Article Info \\
\hline Article history: \\
Received Jan 15, 2019 \\
Revised Apr 21, 2019 \\
Accepted Mar 17, 2019 \\
\hline
\end{tabular}

Keywords:

Anxiety and stress

Depression,

Malay language

MDASS-42

Twitter

\begin{abstract}
Depression, anxiety and stress are not trivial conditions applicable for only the weak-hearted. They can be inflicted by anyone of all age groups, gender, race and social status. While some are courageous to acknowledge their condition, others shy away in shame or denial. In this paper, we proposed a "proactive" approach to detecting candidates of depression, anxiety and stress in an unobtrusive manner by tapping into what Malaysians tweet in Malay language. From this preliminary study, we constructed 165 Malay layman terms which describe depression, anxiety or stress as identified in MDASS-42 scale. Since Twitter is an informal platform, construction of Malay layman terms is an essential step to the detection of candidates. Our study on 1,789 Malay tweets discovered 6 Twitter users as potential candidates, having high frequency of tweets with any of the layman terms. We can conclude that using tweets can be useful in unobtrusively detecting candidates of depression, anxiety or stress. This paper also identifies open research areas.
\end{abstract}

Copyright $(0) 2019$ Institute of Advanced Engineering and Science. All rights reserved.

\section{Corresponding Author:}

Ruhaila Maskat,

Faculty of Computer and Mathematical Sciences,

Universiti Teknologi MARA,

Shah Alam, Selangor, Malaysia.

Email: ruhaila@tmsk.uitm.edu.my

\section{INTRODUCTION}

Mental illness has been pervasive, with depression a standout among psychological problems. It has been reported that mental illness will be the second biggest medical issue inflicting Malaysians after heart disease by 2020 [1]. Untreated depression may expand the possibility of unsafe practices occurring, such as suicide and self-causing injuries. It can affect anyone regardless of age, race, religion or status. Despite the fact that methods of diagnosing psychological problems have progressed over the years, numerous cases still stay undetected [2]. These include depression, anxiety and stress. Current practice in detecting depression is often through psychological assessments intended in establishing a finding or in concluding a psychological assessment such as Hamilton Rating Scale for Depression (HAMD) [3], Montgomery-Åsberg Depression Rating Scale (MADRS) [4], Hospital Anxiety and Depression Scale (HADS) [5], Edinburgh Postnatal Depression Scale (EPDS) [6], Geriatric Depression Scale (GDS) [7], Depression-Anxiety-Stress Scale (DASS) [8] and Malay Depression-Anxiety-Stress Scale (M-DASS) [9]. Each assessment has a specific purpose and target group. HAMD has 21 questions and the scale includes anxiety symptoms to measure just depressive states. Meanwhile, MADRS focuses on measuring only major symptoms of depression and has a scale of 9 questions. HADS contains 14 questions and aims at measuring depression and anxiety states of clinical patients. EPDS, having 10 questions, measures postnatal depression. GDS is used to detect the depression levels of elders, consisting of 15 or 30 questions. DASS detects depression, anxiety and stress levels through 21 or 42 questions. It is not specific to a particular target group and it can be freely 
administered. A Malay language version of DASS [9] was proposed and validated, namely M-DASS-21 [9] and M-DASS-42 [10]. However, for these assessments to be fully utilised they require the deliberate participation of a potentially mentally-ill person. Rodrigues and his team [11] conducted interviews with sufferers and concluded that stigma plays a huge role in affecting the decisions of reaching for help. While some people may not realize they need help, others may deliberately deny they require assistance due to shame since mentally-unwell people in many communities are often shunned. Besides that, sufferers may deny their condition. As a result, mental illness goes undetected and would only be known when it is too late [12].

We hypothesise that social media text can be used to detect candidates of depression, anxiety and stress. Approximately 500 million tweets are posted daily [13]. This phenomenon gives an opportunity for psychologists to get additional data through users' Twitter posts as Twitter contains a considerable amount of information. People have rapidly embraced online communication as a necessary part of day to day life. Individuals even share thoughts online that they may not share in real life [14]. The manner in which individuals write gives a window into their innermost feelings [15]. A "proactive" approach may help to recognize mental illness sufferers or generally potential individuals through the vast scale passive observation of social media status. We suggested two steps in this paper. They are layman terms construction and tweet extraction. This led us to two challenges: semantic and syntax. Semantic requires the identification of layman terms commonly used by tweeters while syntax requires the identification of these terms through their spellings. Twitter is an informal media without the control of an authorized body such as the Dewan Bahasa and Pustaka (DBP) [16] to outline the usage and spelling of the Malay language. Hence, the terms used are often found in everyday conversations which covers a wide range of vocabulary. The richer our list of layman terms is the more likely we would be able to capture potential candidates. Extracting tweets is not a trivial matter. More so in a language like Malay which has been identified as restricted in resources [17]. Differing from the formal Malay language in terms of spelling and sentence construction, Malay social media text has been identified to have spelling variations, sentences with a mix of English and Malay, English words spelt using Malay convention, words based on regional Malaysian slang, words without vowels and words to indicate emphasis [17]. This work aims at detecting candidates of depression, anxiety and stress through Malay-written tweets based on the M-DASS-42 framework.

A growing number of works of literature can be found on the health issues in the context of social media. Stankevich and team [18] conducted a study of depression detection on 887 Reddit users. The goal was to group them into the depressed group and the non-depressed group. To do that, they extracted a few numbers of feature sets. They managed to classify Reddit users with an accuracy score of 90.77\%. [19] meanwhile conducted a study to detect any drug names mentioned in 9852 tweets from Twitter. They obtained an F-Score of $88.64 \%$ in classifying tweets into either "presence of drug-name" or "absence of drug-name". Orabi et.al. [20] tried to detect potential depressed-Twitter users. They obtained 1145 Twitter users where the users were labelled normal, Post-Traumatic Stress Disorder (PTSD) and depressed. The symptoms of depression used in the study were collected from a study by Jamil et al [21]. In the end, they correctly identified Twitter users at an accuracy value of 87.957. Similarly, a study by Shen et al [22] tried to predict depressed users. They extracted roughly 300,000 depressed tweets, 10-billion non-depressed tweets and 3.5 million depression candidate tweets. Then, feature extraction was performed which consists of social network feature, user profile feature, visual feature, emotional feature, topic level feature and domainspecific feature. It was found that they managed to classify depressed, non-depressed and potential depressed users with F-score of $85 \%$. On the other hand, Primack et al. [23] tried to find the correlation between social media platforms and depression and anxiety. They first assessed 1768 individuals using Patient-Reported Outcomes Measurement Information System (PROMIS) [24]. Then the individuals were asked to report their social media accounts, if they were willing to share one. In the end, they managed to obtain social media platforms of Facebook, Twitter, Reddit, Instagram, Snapchat and Tumblr to find the association between these platforms and depression and anxiety. The results showed that social media platforms are independently-associated with depression and anxiety symptoms. Lastly, [25] examined depression symptoms from Twitter and blogs. Roughly 54-million tweets from Twitter and information from blogs related to depression were extracted. The work intended to look for the frequency of words, similarities of words and clustering of words. The results produced that "drink" and "therapy" were the most frequent words used in Twitter and "aggression" from depression blogs. Based on the works of literature, we could definitely rely on social media platforms or specifically Twitter to find potential depressed, anxious and stressed Twitter users.

This paper presents the result of our preliminary study on detecting candidates of depression, anxiety and stress from Twitter posts. An essential step in our methodology is the construction of a list of Malay layman terms based on M-DASS-42 scale's description of depression, anxiety and stress. From the frequency of the terms, we found 6 potential candidates. This detection of candidates can help mitigate a 
tragic result. However, more work is still required to better this method. Open research areas are also identified in this paper.

\section{METHODOLOGY}

Depression, Anxiety and Stress Candidate Detection as shown in Figure 1.

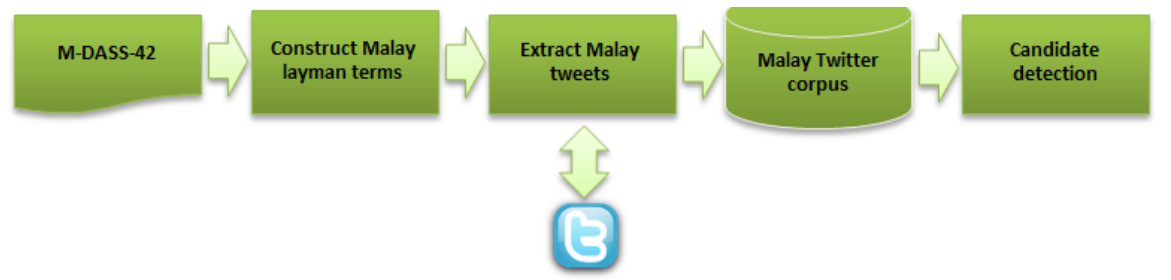

Figure 1. Depression, anxiety and stress candidate detection

\subsection{Malay Layman Terms Construction}

M-DASS-42 has 14 questions for every subscale of depression, anxiety and stress. Each question has a key descriptor e.g. kesal/marah. However, Twitter is an informal platform, densed with layman words and phrases. In order to detect candidates, it is essential that the layman words of these descriptors be identified. Synonymous words from Internet searches and our knowledge as Malay speakers were utilised. Examples can be found in Table 1. Bolded words or phrases are the descriptors. So far, we were able to collect 165 Malay layman terms with 70 indicating depression, 46 anxiety and 49 stress Figure 2.

Table 1. Examples of M-DASS-42 Layman Terms based

\begin{tabular}{ccc}
\hline Subscale & M-DASS-42 & Layman Terms \\
\hline Depression & Saya dapati diri saya menjadi kesal/marah disebabkan & perkara-perkara yang kecil. \\
Anxiety & Saya rasa macam nak pengsan. & $\begin{array}{c}\text { Saya rasa bengkek/bengang/geram/panas hati } \\
\text { pasal benda kecil. }\end{array}$ \\
Stress & Saya dapati sukar untuk bertenang setelah sesuatu \\
& membuatkan saya kesal. & $\begin{array}{c}\text { pitam. } \\
\text { minar/macam nak }\end{array}$ \\
& & $\begin{array}{c}\text { Saya rasa susah nak relaks/susah hati bila buat } \\
\text { benda yang tak patut. }\end{array}$ \\
\hline
\end{tabular}

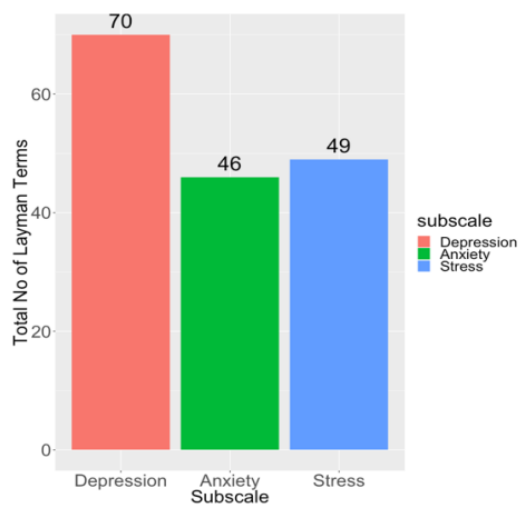

Figure 2. Total number of layman terms constructed per each subscale

\subsection{Candidate Detection}

To detect potential sufferers of depression, anxiety and stress, we extracted tweets from as early as 2010 till February 2019 using Twitter API. Information extracted are the username, tweet, date and time posted and geo-location. However, very little information on geo-location was shared. Only tweets containing our layman terms were collected to reduce the search space. This resulted in the collection of 1,789 tweets. We expect from our observation to detect a user who tweeted a high frequency of these layman 
terms over a period of several weeks or more. This implies that he/she is experiencing prolonged depression, anxiety or stress and thus a candidate. This timeframe is congruent with conditions set by DASS which uses weeks in its assessment to determine a sufferer. The largest number of tweets were found between 2018 and 2019. A breakdown shows that depression descriptors were most tweeted, followed by stress and anxiety Figure 4. Confirmed by a psychologist, Prof Dr Ramli Musa, this pattern of gradual increase across all three subscales has been seen in other earlier works on depression, anxiety and stress involving the voluntary participation of patients. Frequency of tweets across the years 2010 till 2019 as shown in Figure 3.

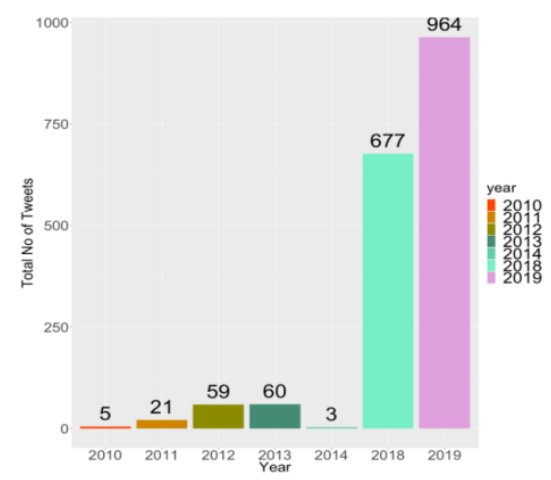

Figure 3. Frequency of tweets across the years 2010 till 2019
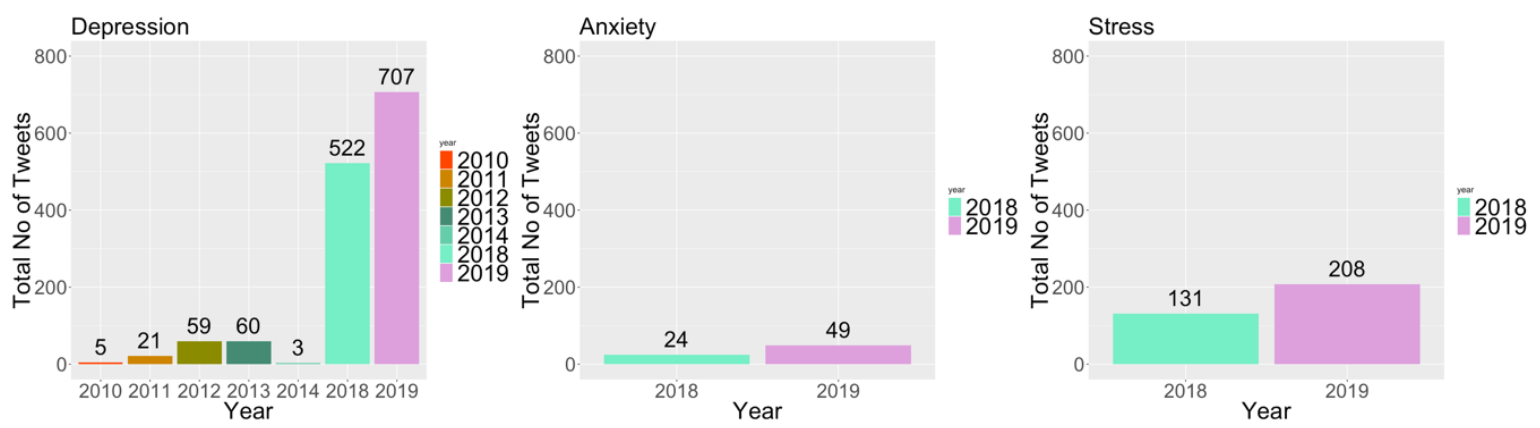

Figure 4. Frequency of tweets against the different subscale of depression, anxiety and stress over the years 2010 till 2019

\section{RESULT}

From our corpus, we calculated the frequency of layman terms found per Twitter user. We discovered 6 users have expressed more than one or more of our layman terms in their tweet Figure 5. We refer to them as mentions. We did not consider the length of interval between the mentions but instead the total mentions within a year. No gender information was provided, hence, a gender-identification process has to be conducted through the author's manner of writing. This is an open research problem for Malay text. In this study, the highest number of mentions is 32 with the lowest is 6 and an average of 19. How significant these values are in terms of the levels of severity is also an open area for further research. To use the current DASS severity formula Table 2 would not yield meaningful results, the reason being DASS has a set boundary described by 21 or 42 conditions, depending on the DASS version. Thus, severity levels can be calculated in relation to the boundary based on the percentile. For example, if a patient exhibits all 42 conditions, therefore, the severity level means extremely severe (100 percentile). On the other hand, if only 3 conditions were exhibited, it can be concluded that the severity level is normal ( $<78$ percentile). Figure 6 shows a breakdown of the frequency per user and the layman terms that were mentioned. Our result shows that all 5 users repeatedly tweeted about conditions of stress and only 1 user on depression. When combined with results in Figure 4, we found that many Malaysians experience depression and a few can be substantially more than others, whereas stress effects a smaller number of Malaysians but can be considerable.

This method helped us to identify candidate sufferers, however, other descriptor selection methods need to be introduced besides only synonyms to increase detection coverage. Impeding effective detection of 
candidates is the syntactic nature of the Malay tweets which are laden with characteristics described in [12]. They are spelling variations, Malay-English mix sentence, Malay-spelling English words, slang-based words, vowel-les words, emphasis words. We also discovered that the attribute of geo-location is hardly made available to the public by a majority of Twitter users. Figure 7 shows the frequency of users not providing specific information regarding their geo-location. Based on the figure, it can be seen that out of 1,789 tweets, only 3 tweets shared their geo-locations. This makes it impossible to perform further analysis relating to geolocation. Geo-location is a useful piece of information as a next step to prevent sufferers from reckless decisions, where in a worst-case scenario, physically intercepting something tragic like a suicide attempt. It can also help in discovering hotspots of depression, anxiety and stress, allowing preventive efforts to take place. A possible alternative to the lack of this information is to identify the regional slang used to write a tweet, if any. This, however, is less precise due to people's mobility from one Malaysia state to another and the large area that a region span.

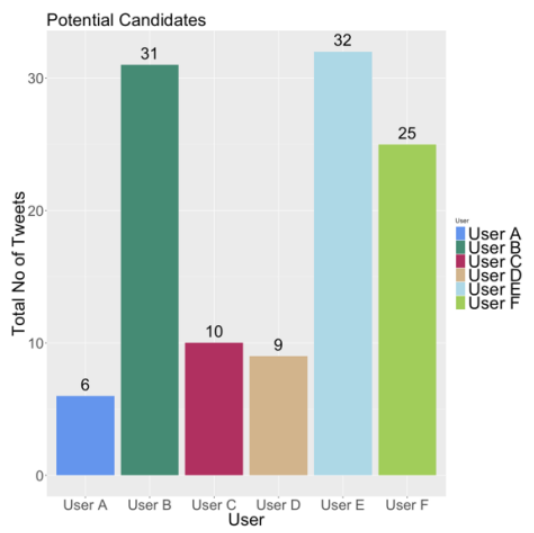

Table 2. DASS Scoring

\begin{tabular}{cccccc}
\hline & Depression & Anxiety & Stress & Z-score & Percentile \\
\hline Normal & $0-9$ & $0-7$ & $0-14$ & $<0.5$ & $<78$ \\
Mild & $10-13$ & $8-9$ & $15-18$ & 0.5 to 1.0 & 78 to 87 \\
Moderate & $14-20$ & $10-14$ & $19-25$ & $>1.0$ to 2.0 & $>87$ to 95 \\
Severe & $21-27$ & $15-19$ & $26-33$ & $>2.0$ to 3.0 & $>95$ to 98 \\
Extremely Severe & 28 & $20+$ & $34+$ & $>3.0$ & $>98$ to 100 \\
\hline
\end{tabular}

Figure 5. Frequency of

Twitter Users as Possible Candidates
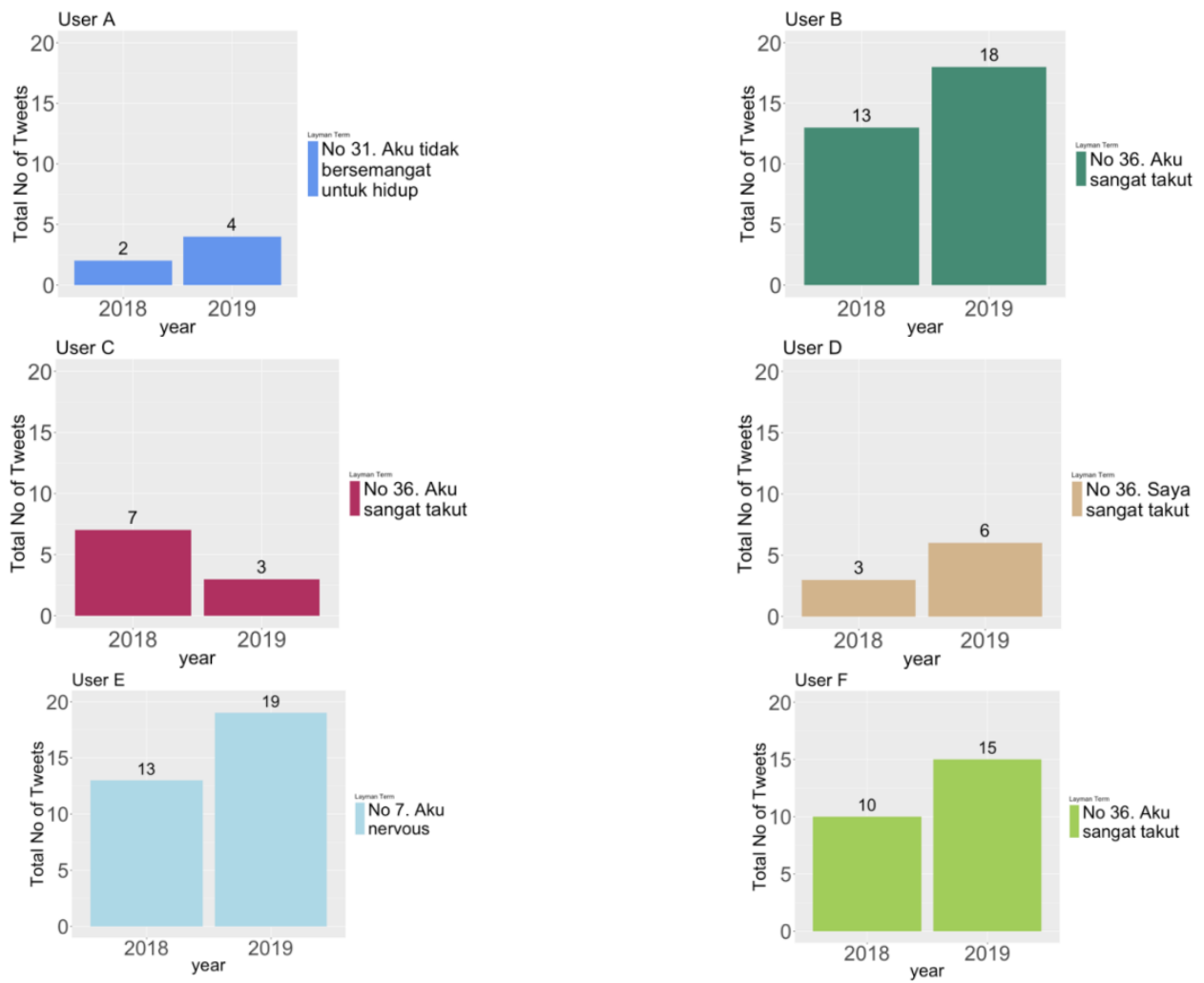

Figure 6. Frequency and layman term used over the period of time it was tweeted 


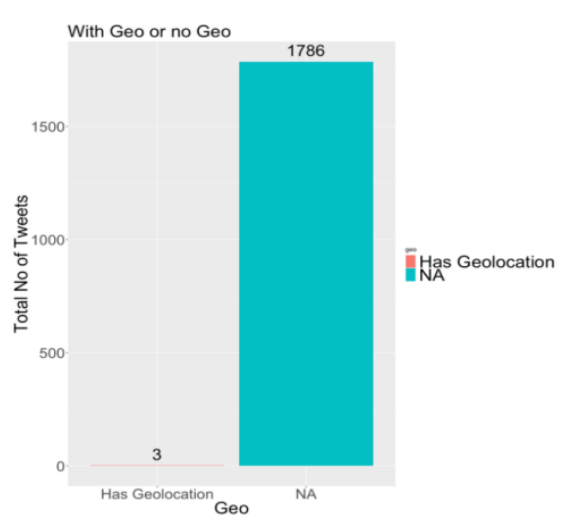

Figure 7. Frequency of users not providing geo-location

\section{CONCLUSION}

We proposed a proactive and unobtrusive approach to detecting candidates of depression, anxiety and stress from Malay-written tweets. The cornerstone of this approach is the construction of Malay layman terms to help in the detection. In this preliminary study, we were able to discover 6 potential sufferers of depression, anxiety and stress. Further work would be to propose an approach to produce a rich vocabulary of Malay layman terms quickly, to deal with the syntactic challenges of Malay's social media and to investigate how a new scale to calculate the levels of severity can be introduced when detecting through tweets.

\section{ACKNOWLEDGEMENTS}

Our acknowledgement goes to the Faculty of Computer and Mathematical Sciences, Universiti Teknologi MARA Shah Alam, Malaysia and the Department of Psychiatry, Kulliyyah of Medicine, International Islamic University MalaysiaInternational Islamic University Malaysia for the support given.

\section{REFERENCES}

[1] Mustapha, W.N.W., "Depression on the rise," New Straits Times, April 24, 2007. [Online], Available: https://www.nst.com.my/opinion/columnists/2018/04/361026/depression-rise [Accessed Feb. 7, 2019].

[2] Guntuku, S.C., Yaden D.B., Kern M.L., Ungar L.H, Eichstaedt J.C. "Detecting depression and mental illness on social media: an integrative review," Current Opinion in Behavioral Sciences. vol. 18, pp. 43-9, 2017.

[3] Hamilton, M. "The Hamilton rating scale for depression," In Assessment of depression 1986, Springer, (pp. 143-152).

[4] Montgomery, S.A., Åsberg M.A. "A new depression scale designed to be sensitive to change," The British journal of psychiatry, vol. 134(4), pp. 382-9, 1979.

[5] Zigmond, A.S., Snaith RP. "The hospital anxiety and depression scale," Acta psychiatrica scandinavica. vol. 67(6), pp. 361-70, 1983.

[6] Cox, J.L., Holden, J.M., Sagovsky, R. "Detection of postnatal depression: development of the 10-item Edinburgh Postnatal Depression Scale," The British journal of psychiatry, vol. 150(6), pp. 782-6, 1987.

[7] Sheikh, J.I,. Yesavage, J.A. "Geriatric Depression Scale (GDS): recent evidence and development of a shorter version," Clinical Gerontologist: The Journal of Aging and Mental Health. 1986 Jun.

[8] Lovibond, S.H. "The nature and measurement of anxiety, stress and depression," In 18th Annual Conference of the Australian Psychological Society, University of Western Australia, May 1983 May.

[9] Musa, R., Fadzil, M.A., Zain, Z., "Translation, validation and psychometric properties of Bahasa Malaysia version of the Depression Anxiety and Stress Scales (DASS)," ASEAN Journal of Psychiatry, vol. 8(2), pp. 82-9, 2007.

[10] Ramli ,M., Rosnani S, A.R.A.F. "Psychometric profile of malaysian version of the Depressive, Anxiety and Stress Scale 42-item (DASS-42)," Malaysian Journal of Psychiatry, vol. 21(1), 2012.

[11] Rodrigues, S. et.al, "Impact of stigma on veteran treatment seeking for depression," American Journal of Psychiatric Rehabilitation, vol. 17(2), pp. 128-46, 2014.

[12] The Ranch, "The Myth of Denial: Why People With Mental Health Disorders Refuse Treatment," 2019 [Online]. Available:https://www.recoveryranch.com/mental-health/the-myth-of-denial-why-people-with-mental-healthdisorders-refuse-treatment/.

[13] Omnicore, "Twitter by the Numbers: Stats, Demographics \& Fun Facts," Jan 6. [Online]. Available: https://www.omnicoreagency.com/twitter-statistics/. [Accessed Feb 7, 2019].

[14] Digital Influence Lab, "Malaysia Digital Marketing Statistics 2017" 2017 [Online]. Available: http://digitalinfluencelab.com/malaysia-digital-marketing-stats/. [Accessed Feb 7, 2019]. 
[15] Seiter C, "The Psychology of Social Media: Why We Like, Comment, and Share Online,"Jan 18. [Online]. Available: https://buffer.com/resources/psychology-of-social-media. [Accessed Feb 7, 2019]

[16] Dewan Bahasa dan Pustaka, 2019 [Online]. Available: http://lamanweb.dbp.gov.my/

[17] Maskat, R., Munarko, Y. "A Taxonomy of Malay Social Media Text," International Conference on Engineering and Computing, 2019.

[18] Stankevich, M., Isakov, V., Devyatkin D., Smirnov I. "Feature Engineering for Depression Detection in Social Media," In ICPRAM, pp. 426-431, 2018.

[19] Tokala, S., Gambhir, V., Mukherjee, A., "Deep Learning for social media health text classification," In Proceedings of the 2018 EMNLP Workshop SMM4H: The 3rd Social Media Mining for Health Applications Workshop and Shared Tas, pp. 61-64, 2018.

[20] Orabi, A.H, Buddhitha, P., Orabi M.H., Inkpen D. "Deep Learning for Depression Detection of Twitter Users," In Proceedings of the Fifth Workshop on Computational Linguistics and Clinical Psychology: From Keyboard to Clinic, pp. 88-97, 2018.

[21] Jamil, Z. Monitoring tweets for depression to detect at-risk users (Doctoral dissertation, Université d'Ottawa/University of Ottawa).

[22] Shen, G. et.al. "Depression Detection via Harvesting Social Media: A Multimodal Dictionary Learning Solution. In IJCAI, pp. 3838-3844, 2017.

[23] Primack, B.A. et al. "Use of multiple social media platforms and symptoms of depression and anxiety: A nationally-representative study among US young adults," Computers in human behavior, vol. 69, pp. 1-9, 2017.

[24] Cella, D et.al. "The Patient-Reported Outcomes Measurement Information System (PROMIS): progress of an NIH Roadmap cooperative group during its first two years, Medical care, vol. 45(5 Suppl 1):S3, 2007.

[25] Ma, L., Wang, Z., Zhang, Y. "Extracting Depression Symptoms from Social Networks and Web Blogs via Text Mining," In International Symposium on Bioinformatics Research and Applications, Springer, pp. 325-330, 2017.

\section{BIOGRAPHIES OF AUTHORS}

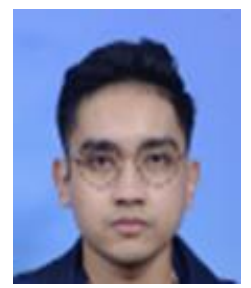

Muhammad Zahier Nasrudin is a student of Master in Data Science at Universiti Teknologi Mara (UiTM) where he received dean's list award in the first semester. He previously received a Bachelor's Degree in Actuarial Science from UiTM in 2016. Zahier and his basketball team won a silver medal in interfaculty sports championship.

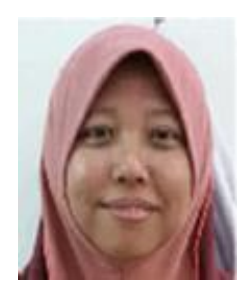

Ruhaila Maskat received her PhD in Computer Science from the University of Manchester, United Kingdom. She is a Senior Lecturer at the Universiti Teknologi MARA Shah Alam, Malaysia where she teaches courses related to Data Analytics. Her main research interest is in big data analytics, text analytics, social media analytics, data mining and data integration.

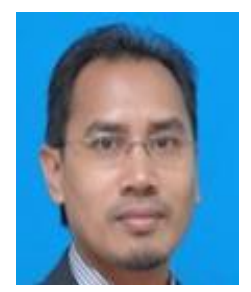

Ramli Musa is a professor of psychiatry and a consultant psychiatrist at the Department of Psychiatry, International Islamic University Malaysia. Apart from his teaching and clinical duties, he is also active in research and social community works. He established a database of validated scales in Malaysia and received many accolades related to designing scales and IT based research. 